\title{
BURKHARDT QUARTIC, BARTH SEXTIC, AND THE ICOSAHEDRON
}

\author{
IVAN CHELTSOV, VICTOR PRZYJALKOWSKI, AND CONSTANTIN SHRAMOV
}

\begin{abstract}
We study two rational Fano threefolds with an action of the icosahedral group $\mathfrak{A}_{5}$. The first one is the famous Burkhardt quartic threefold, and the second one is the double cover of the projective space branched in the Barth sextic surface. We prove that both of them are $\mathfrak{A}_{5}$-Fano varieties that are $\mathfrak{A}_{5}$-birationally superrigid. This gives two new embeddings of the group $\mathfrak{A}_{5}$ into the space Cremona group.
\end{abstract}

\section{INTRODUCTION}

One of the interesting subjects in three-dimensional birational geometry is studying $G$-Fano threefolds (see $[\operatorname{Pr} 13]$ ), where $G$ is a finite group. These are Fano threefolds with terminal singularities such that the rank of their $G$-invariant class group equals 1 . Finding enough details about their $G$-equivariant (biregular) geometry often leads to conclusions about the absence of $G$-equivariant birational maps between them, see e.g. CS14 and CS15]. It is a usual case that on this way one encounters really beautiful geometric constructions arising from large groups of symmetries of the corresponding varieties.

The icosahedral group $\mathfrak{A}_{5}$ appears as a group of symmetries for a remarkably large class of Fano threefolds, and many of them are $\mathfrak{A}_{5}$-Fano threefolds, see e.g. [CS15], CPS16], and [PS16]. In this paper we study two rational Fano threefolds with an action of the group $\mathfrak{A}_{5}$. Both of these threefolds are singular and have only nodes as singularities; moreover, they are "extremal" in some sense, which makes their geometry more interesting. We prove that the ranks of their $\mathfrak{A}_{5}$-invariant class groups equal 1 , so that both of them are $\mathfrak{A}_{5}$-Fano threefolds. We use this to prove that they are $\mathfrak{A}_{5}$-birationally superrigid, see [CS15, §3.1] for a definition.

Consider the projective space $\mathbb{P}^{5}$ with homogeneous coordinates $x_{0}, \ldots, x_{5}$. Denote by $\sigma_{k}\left(x_{0}, \ldots, x_{5}\right)$ the $k$-th elementary symmetric polynomial in $x_{0}, \ldots, x_{5}$. The Burkhardt quartic is defined in $\mathbb{P}^{5}$ by equations

$$
\sigma_{1}\left(x_{0}, \ldots, x_{5}\right)=\sigma_{4}\left(x_{0}, \ldots, x_{5}\right)=0 .
$$

This quartic threefold was first described by Burkhardt in [Bu91]. It has 45 nodes, which is actually the largest possible number of isolated singularities for quartic threefolds, see [JS-BV90] (cf. [Pr17]).

The Burkhardt quartic is known to be rational (see To35]), and its automorphism group is isomorphic to $\mathrm{PSp}_{4}\left(\mathbf{F}_{3}\right)$, see [Co06, §6] and [JS-BV90]. Let the group $\mathfrak{S}_{6}$ act on $\mathbb{P}^{5}$ by permutations of homogeneous coordinates $x_{0}, \ldots, x_{5}$. Then $\mathfrak{S}_{6}$ preserves the Burkhardt quartic.

Let us refer to a subgroup $\mathfrak{A}_{5}$ in $\mathfrak{S}_{6}$ that fixes one of the homogeneous coordinates $x_{0}, \ldots, x_{5}$ as a standard subgroup $\mathfrak{A}_{5}$, and to a subgroup $\mathfrak{A}_{5}$ of $\mathfrak{S}_{6}$ that does not fix any of these coordinates as a non-standard subgroup $\mathfrak{A}_{5}$. Our first result is

Theorem 1.1. Let $G$ be a standard subgroup $\mathfrak{A}_{5}$ in $\mathfrak{S}_{6}$. The Burkhardt quartic is a rational $G$-Fano threefold that is $G$-birationally superrigid.

It should be pointed out that if $G^{\prime}$ is a non-standard subgroup $\mathfrak{A}_{5}$ in $\mathfrak{S}_{6}$, then the Burkhardt quartic is not a $G^{\prime}$-Fano threefold (see Remark 2.12 below). 
Now consider the projective space $\mathbb{P}^{3}$ that is a projectivization of a non-trivial reducible fourdimensional representation of the group $\mathfrak{A}_{5}$. There exists a unique $\mathfrak{A}_{5}$-invariant sextic surface $\mathcal{B}$ with 65 nodes in $\mathbb{P}^{3}$, which is actually the largest possible number of nodes for a sextic surface, see [JR97]. This surface was discovered by Barth in [Ba96] and is usually called the Barth sextic. Our second result is

Theorem 1.2. The double cover of $\mathbb{P}^{3}$ branched over the surface $\mathcal{B}$ is a rational $\mathfrak{A}_{5}$-Fano threefold that is $\mathfrak{A}_{5}$-birationally superrigid.

Theorems 1.1 and 1.2 give two embeddings of the group $\mathfrak{A}_{5}$ into the space Cremona group that are not conjugate to each other. Moreover, these embeddings are also not conjugate to any of the four embeddings described in [CS15, Remark 1.2.1], CS15, Example 1.3.9], [CS15, Theorem 1.4.1], and [CPS16, Theorem 4.2].

Notation and conventions. All varieties are defined over the field $\mathbb{C}$ of complex numbers. By $\mathrm{Cl}(X)$ we denote the group of linear equivalence classes of Weil divisors on a variety $X$. If a variety $V$ is acted on by a finite group $G$, and $Z$ is a subvariety of $V$, we will sometimes abuse terminology and refer to the union of the images $g(Z), g \in G$, as the $G$-orbit of $Z$. By a node we always mean an isolated singularity that is locally isomorphic to the singularity of a cone over a smooth quadric of the appropriate dimension.

Acknowledgements. We are grateful to A.Kuznetsov, D. Pasechnik, V.Popov, Yu. Prokhorov, and L. Rybnikov for useful discussions. The study has been funded by the Russian Academic Excellence Project "5-100". V. Przyjalkowski was partially supported by Laboratory of Mirror Symmetry NRU HSE, RF Government grant, ag. 14.641.31.0001. C. Shramov was also supported by the grants RFFI 15-01-02158, RFFI 15-01-02164, RFFI 14-01-00160, and by Dynasty foundation.

\section{Burkhardt QUARTIC}

In this section we prove Theorem 1.1. Recall that the Burkhardt quartic $X$ is given in the projective space $\mathbb{P}^{5}$ with homogeneous coordinates $x_{0}, \ldots, x_{5}$ by equations

$$
\sigma_{1}\left(x_{0}, \ldots, x_{5}\right)=\sigma_{4}\left(x_{0}, \ldots, x_{5}\right)=0,
$$

where $\sigma_{k}\left(x_{0}, \ldots, x_{5}\right)$ is the $k$-th elementary symmetric polynomial in $x_{0}, \ldots, x_{5}$. The quartic $X$ is rational, and its automorphism group is isomorphic to $\operatorname{PSp}_{4}\left(\mathbf{F}_{3}\right)$. A subgroup $\mathfrak{S}_{6} \subset \operatorname{PSp}_{4}\left(\mathbf{F}_{3}\right)$ that acts by permutations of the homogeneous coordinates $x_{0}, \ldots, x_{5}$ preserves $X$. The Burkhardt quartic has 45 singularities that form two $\mathfrak{S}_{6}$-orbits: one is the $\mathfrak{S}_{6}$-orbit of length 30 of the point

$$
\left[1: 1: \omega: \omega: \omega^{2}: \omega^{2}\right]
$$

and the other is the $\mathfrak{S}_{6}$-orbit of length 15 of the point

$$
[1:-1: 0: 0: 0: 0] \text {. }
$$

Remark 2.1 (cf. $\left[\mathrm{C}^{+}\right.$, p. 26]). Up to conjugation, the group $\operatorname{PSp}_{4}\left(\mathbf{F}_{3}\right)$ contains a unique subgroup isomorphic to $\mathfrak{S}_{6}$, and two subgroups isomorphic to $\mathfrak{A}_{5}$. The latter agree with two non-conjugate embeddings of $\mathfrak{A}_{5}$ to $\mathfrak{S}_{6} \subset \operatorname{PSp}_{4}\left(\mathbf{F}_{3}\right)$.

Lemma 2.2. One has $\operatorname{rk} \operatorname{Cl}(X)^{\mathfrak{S}_{6}}=1$.

Proof. It is well-known that the quotient $\mathbb{P}^{5} / \mathfrak{S}_{6}$ is isomorphic to the weighted projective space $\mathbb{P}(1,2,3,4,5,6)$, so that

$$
\mathbb{P}^{4} / \mathfrak{S}_{6} \cong \mathbb{P}(2,3,4,5,6)
$$

and $X / \mathfrak{S}_{6} \cong \mathbb{P}(2,3,5,6)$. In particular, one has $\operatorname{rk} \mathrm{Cl}(X)^{\mathfrak{S}_{6}}=\operatorname{rk} \mathrm{Cl}\left(X / \mathfrak{S}_{6}\right)=1$. 
Corollary 2.3. One has $\mathrm{rk} \mathrm{Cl}(X)^{\mathrm{PSp}_{4}\left(\mathbf{F}_{3}\right)}=1$.

If one ignores the action of the automorphism group of $X$, the following result is known.

Lemma 2.4 ([Ka11, Theorem 1.1(iii)]). One has $\operatorname{rk} \mathrm{Cl}(X)=16$.

We are going to find the ranks of the invariant parts of $\mathrm{Cl}(X)$ with respect to various groups. Consider the vector space

$$
\mathrm{Cl}(X)_{\mathbb{C}}=\mathrm{Cl}(X) \otimes \mathbb{C}
$$

as a representation of the group $\operatorname{PSp}_{4}\left(\mathbf{F}_{3}\right)$. Then $\mathrm{Cl}(X)_{\mathbb{C}}$ contains a trivial subrepresentation $\mathbb{K}$ corresponding to the canonical class, and one has $\mathrm{Cl}(X)_{\mathbb{C}} \cong \mathbb{K} \oplus \mathbb{W}$. We know from Lemma 2.4 that $\mathbb{W}$ is a 15-dimensional representation of $\operatorname{PSp}_{4}\left(\mathbf{F}_{3}\right)$. Obviously, $\mathbb{W}$ is defined over the field $\mathbb{Q}$.

Lemma 2.5. The complex $\mathrm{PSp}_{4}\left(\mathbf{F}_{3}\right)$-representation $\mathbb{W}$ is irreducible.

Proof. Recall that all non-trivial irreducible representations of $\operatorname{PSp}_{4}\left(\mathbf{F}_{3}\right)$ of dimension less than 15 are two 5 -dimensional representations $\chi_{2}$ and $\chi_{3}$ that are not defined over $\mathbb{Q}$, one 6-dimensional representation $\chi_{4}$, and two 10-dimensional representations $\chi_{5}$ and $\chi_{6}$ that are not defined over $\mathbb{Q}$ (see $\left[\mathrm{C}^{+}\right.$, p. 27]).

Suppose that $\mathbb{W}$ is reducible. Note that $\mathbb{W}$ does not have trivial subrepresentations by Corollary 2.3. Therefore, $\mathbb{W}$ splits as a sum of representations of $\mathrm{PSp}_{4}\left(\mathbf{F}_{3}\right)$ isomorphic to $\chi_{2}, \chi_{3}, \chi_{4}$, $\chi_{5}$, or $\chi_{6}$. However, keeping in mind that $\operatorname{dim}(\mathbb{W})=15$ we see that there are no summands isomorphic to $\chi_{4}$. Moreover, since $\chi_{5}$ and $\chi_{6}$ are not defined over $\mathbb{Q}$, they either appear as summands in $\mathbb{W}$ simultaneously, or do not appear at all. The same holds for $\chi_{2}$ and $\chi_{3}$. An obtained contradiction completes the proof of the lemma.

By Lemma 2.5, the $\operatorname{PSp}_{4}\left(\mathbf{F}_{3}\right)$-representation $\mathbb{W}$ is isomorphic to one of the two 15-dimensional irreducible representations of $\operatorname{PSp}_{4}\left(\mathbf{F}_{3}\right)$ (see $\left[\mathrm{C}^{+}\right.$, p. 27]). In the notation of $\left[\mathrm{C}^{+}\right.$, p. 27] these are $\chi_{7}$ and $\chi_{8}$. In fact, we have $\mathbb{W} \cong \chi_{7}$. This follows from Lemma 2.2 and (the first part of) the following result.

Lemma 2.6. The following assertions hold:

(i) the $\mathfrak{S}_{6}$-representation $\left.\chi_{7}\right|_{\mathfrak{S}_{6}}$ does not contain trivial subrepresentations, while the $\mathfrak{S}_{6}$ representation $\left.\chi_{8}\right|_{\mathfrak{S}_{6}}$ does;

(ii) for one of the two non-conjugate embeddings of $\mathfrak{A}_{5}$ to $\operatorname{PSp}_{4}\left(\mathbf{F}_{3}\right)$, the $\mathfrak{A}_{5}$-representation $\left.\chi_{7}\right|_{\mathfrak{A}_{5}}$ does not contain trivial subrepresentations, while for the other embedding it contains a unique trivial subrepresentation.

Proof. Both assertions are obtained by direct computations. We used the GAP software GAP] to perform them.

Note that Lemma 2.6(ii) implies that the invariant part of $\mathrm{Cl}(X)$ with respect to one of the two non-conjugate actions of $\mathfrak{A}_{5}$ on $X$ has rank 1 .

Later we will need the following elementary result.

Lemma 2.7. Let $Y$ be a normal variety acted on by a finite group $G$. Suppose that there exist Weil divisors $\Pi_{1}, \ldots, \Pi_{r}$ on $Y$ such that they generate the $\mathbb{Q}$-vector space

$$
\mathrm{Cl}(Y)_{\mathbb{Q}}=\mathrm{Cl}(Y) \otimes \mathbb{Q},
$$

and $\Pi_{1}, \ldots, \Pi_{r}$ form one $G$-orbit. Then the $\mathbb{Q}$-vector space

$$
\mathrm{Cl}(Y)_{\mathbb{Q}}^{G}=\mathrm{Cl}(Y)^{G} \otimes \mathbb{Q}
$$

is one-dimensional. 
Proof. Let $D$ be a $G$-invariant Weil divisor on $Y$. By assumption, we have

$$
D \sim_{\mathbb{Q}} \sum_{i=1}^{r} a_{i} \Pi_{i}
$$

for some rational numbers $a_{1}, \ldots, a_{r}$. Put $\mathcal{P}=\Pi_{1}+\ldots+\Pi_{r}$. Then

$$
|G| D \sim \sum_{g \in G} g(D) \sim_{\mathbb{Q}} \sum_{i=1}^{r} a_{i} \sum_{g \in G} g\left(\Pi_{i}\right)=\sum_{i=1}^{r} a_{i} \frac{|G|}{r} \mathcal{P}=\frac{|G|}{r}\left(\sum_{i=1}^{r} a_{i}\right) \mathcal{P} .
$$

In particular, we see that the $\mathbb{Q}$-vector space $\mathrm{Cl}(Y)_{\mathbb{Q}}^{G}$ is generated by $\mathcal{P}$.

Corollary 2.8. Let $Y$ be a nodal Fano threefold acted on by a finite group $G$, and let $i_{Y}$ be the largest positive integer such that $-K_{Y} \sim i_{Y} H$, where $H$ is an ample Cartier divisor on $Y$. Suppose that there exist Weil divisors $\Pi_{1}, \ldots, \Pi_{r}$ on $Y$ such that they generate the $\mathbb{Q}$-vector space

$$
\mathrm{Cl}(Y)_{\mathbb{Q}}=\mathrm{Cl}(Y) \otimes \mathbb{Q},
$$

and $\Pi_{1}, \ldots, \Pi_{r}$ form one $G$-orbit. Then $\mathrm{Cl}(Y)^{G}=\mathbb{Z} \cdot H$.

Proof. By Lemma 2.7, the $\mathbb{Q}$-vector space $\mathrm{Cl}(Y)_{\mathbb{Q}}^{G}$ is one-dimensional. Since any singular point $O$ of $Y$ is a node, we know that any Weil divisor that is $\mathbb{Q}$-Cartier in a neighborhood of $O$ is actually Cartier in a neighborhood of $O$. In particular, every $G$-invariant Weil divisor on $Y$ is a Cartier divisor. Since the Picard group of $Y$ has no torsion (this holds even for Fano varieties with log terminal singularities, see e.g. [IP99, Proposition 2.1.2]), this implies that $\operatorname{rk} \operatorname{Cl}(Y)^{G}=1$, and the assertion follows.

Now we are ready to start proving Theorem 1.1. Recall that a subgroup $\mathfrak{A}_{5}$ in $\mathfrak{S}_{6}$ that fixes one of the homogeneous coordinates $x_{0}, \ldots, x_{5}$ is called a standard subgroup $\mathfrak{A}_{5}$, and a subgroup $\mathfrak{A}_{5}$ of $\mathfrak{S}_{6}$ that does not fix any of these homogeneous coordinates is called a nonstandard subgroup $\mathfrak{A}_{5}$. By Remark 2.1 subgroups of these two kinds represent two conjugacy classes of subgroups isomorphic to $\mathfrak{A}_{5}$ in $\operatorname{PSp}_{4}\left(\mathbf{F}_{3}\right)$.

Let $G$ be a standard subgroup $\mathfrak{A}_{5}$ in $\mathfrak{S}_{6}$ that fixes the homogeneous coordinate $x_{5}$. We are going to prove the following result.

Proposition 2.9. The group $\mathrm{Cl}(X)^{G}$ is generated by $-K_{X}$.

As a consequence of Proposition 2.9 we can find the rank of the Weil divisor class group invariant under any given group that contains a standard subgroup $\mathfrak{A}_{5}$. In particular, for a subgroup $\mathfrak{A}_{6} \subset \mathfrak{S}_{6}$ we get

Corollary 2.10. One has $\operatorname{rk} \mathrm{Cl}(X)^{\mathfrak{A}_{6}}=1$.

Remark 2.11. The assertion of Corollary 2.10 was used in the proof of [CS14, Theorem 1.20]. Moreover, the authors of [CS14] gave a brief sketch of a proof of this assertion, but this proof was actually incorrect. Indeed, contrary to what was claimed in the proof of [CS14, Theorem 1.20], the quotient of $X$ by a subgroup $\mathfrak{S}_{4} \subset \mathfrak{A}_{6}$ is not isomorphic to the weighted projective space $\mathbb{P}(1,2,2,3)$. Our proof of Corollary 2.10 fixes this gap and thus recovers the proof of [CS14, Theorem 1.20].

Remark 2.12. Let $G^{\prime}$ be a non-standard subgroup $\mathfrak{A}_{5} \subset \mathfrak{S}_{6}$. Then Proposition 2.9 and Lemma 2.6(ii) imply that $\operatorname{rk} \mathrm{Cl}(X)^{G^{\prime}}=2$.

Now we derive Theorem 1.1 from Proposition 2.9. 
Proof of Theorem 1.1. The proof is standard, see [CS14, Theorem 1.20] and [Me04], but we include it for the reader's convenience.

Suppose that $X$ is not $G$-birationally superrigid. Since the divisor $-K_{X}$ generates the group $\mathrm{Cl}(X)^{G}$ by Proposition 2.9, it follows from [CS15, Corollary 3.3.3] that there exist a positive integer $n$ and a $G$-invariant linear subsystem $\mathcal{M}$ of the linear system $\left|-n K_{X}\right|$ such that $\mathcal{M}$ does not have fixed components, and the log pair $\left(X, \frac{1}{n} \mathcal{M}\right)$ is not canonical. Choose two general surfaces $M_{1}$ and $M_{2}$ in the linear system $\mathcal{M}$, and denote by $H$ a general hyperplane section of $X$. Then $H \sim-K_{X}$.

Suppose that there is an irreducible curve $C \subset X$ such that the log pair $\left(X, \frac{1}{n} \mathcal{M}\right)$ is not canonical along $C$. Denote by $Z$ the $G$-orbit of the curve $C$. Put $d=H \cdot Z$ and $m=\operatorname{mult}_{C}\left(M_{1}\right)=\operatorname{mult}_{C}\left(M_{2}\right)$. Then $m>n$, so that

$$
4 n^{2}=M_{1} \cdot M_{2} \cdot H \geqslant d m^{2}>d n^{2},
$$

which gives $d \leqslant 3$. In particular, one has $Z=C$. Since $\mathbb{P}^{4}$ does not contain $G$-invariant lines and planes, we see that $Z$ is a twisted cubic. Moreover, the curve $Z$ is contained in the smooth locus of $X$, because the possible lengths of $G$-orbits in $Z$ are 12, 20,30,60, and there are no $G$-orbits of such lengths consisting of singular points of $X$.

Let $f: W \rightarrow X$ be the blow up along the curve $Z$. Denote by $F$ the $f$-exceptional divisor. Denote by $\widetilde{M}_{1}$ and $\widetilde{M}_{2}$ the proper transforms of the surfaces $M_{1}$ and $M_{2}$ on the threefold $W$, respectively. We then get

$$
\widetilde{M}_{1} \sim \widetilde{M}_{2} \sim f^{*}(n H)-m F .
$$

Moreover, the divisor $f^{*}(2 H)-F$ is nef, because $Z$ is a scheme-theoretic intersection of quadrics. Keeping in mind that $F^{3}=-K_{X} \cdot Z-2=1$ and $H \cdot Z=3$, we obtain

$$
0 \leqslant\left(f^{*}(2 H)-F\right) \cdot \widetilde{M}_{1} \cdot \widetilde{M}_{2}=\left(f^{*}(2 H)-F\right)\left(f^{*}(n H)-m F\right)^{2}=8 n^{2}-6 n m-5 m^{2}<0
$$

because $m>n$. This is a contradiction.

Thus, the log pair $(X, \lambda \mathcal{M})$ is canonical outside of finitely many points of $X$. Take any point $P \in X$ such that the singularities of the log pair $(X, \lambda \mathcal{M})$ are not canonical at the point $P$. Suppose that $P$ is a smooth point of $X$, and let $H_{P}$ be a general hyperplane section of $X$ passing through $P$. Then

$$
4 m=M_{1} \cdot M_{2} \cdot H_{P} \geqslant \operatorname{mult}_{P}\left(M_{1} \cdot M_{2}\right)>4 n^{2}
$$

by [Co00, Corollary 3.4]. The obtained contradiction shows that $X$ is singular at $P$.

Let $\Sigma$ be the $G$-orbit of the point $P$. Then there is a subset $\Gamma \subset \Sigma$ such that $|\Gamma|=4$, and the set $\Gamma$ is not contained in any plane in $\mathbb{P}^{4}$. Let $g: U \rightarrow X$ be a blow up of $\Gamma$, and let $E_{1}, E_{2}$, $E_{3}$, and $E_{4}$ be exceptional divisors of $g$. Denote by $\bar{M}_{1}$ and $\bar{M}_{2}$ the proper transforms of the surfaces $M_{1}$ and $M_{2}$ on the threefold $U$, respectively. We then get

$$
\bar{M}_{1} \sim \bar{M}_{2} \sim g^{*}(n H)-\delta \sum_{i=1}^{4} E_{i}
$$

for some positive integer $\delta$. Moreover, it follows from [Co00, Theorem 3.10] that $\delta>n$. On the other hand, the divisor $g^{*}(2 H)-E_{1}-E_{2}-E_{3}-E_{4}$ is nef, because the points of $\Gamma$ are not coplanar. In particular, we have

$$
0 \leqslant\left(g^{*}(2 H)-\sum_{i=1}^{4} E_{i}\right) \cdot \bar{M}_{1} \cdot \bar{M}_{2}=\left(f^{*}(2 H)-\sum_{i=1}^{4} E_{i}\right)\left(f^{*}(n H)-\delta \sum_{i=1}^{4} E_{i}\right)^{2}=8 n^{2}-8 \delta^{2} .
$$

This is impossible, since $\delta>n$. 
In the rest of this section we give a proof of Proposition 2.9, Recall from [Hu96, §5.2.1] that the Burkhardt quartic $X$ contains forty $j$-planes, that are planes passing through nine singular points of $X$. Let us describe them. For any triple of indices $0 \leqslant i_{1}<i_{2}<i_{3} \leqslant 5$, we denote by $\Pi_{i_{1} i_{2} i_{3}}^{+}$the plane given in $\mathbb{P}^{5}$ by equations

$$
x_{i_{2}}=\omega x_{i_{1}}, \quad x_{i_{3}}=\omega^{2} x_{i_{1}}, \quad \sigma_{1}\left(x_{0}, \ldots, x_{5}\right)=0,
$$

where $\omega$ is a primitive cubic root of 1 , and we denote by $\Pi_{i_{1} i_{2} i_{3}}^{-}$the plane given in $\mathbb{P}^{5}$ by equations

$$
x_{i_{3}}=\omega x_{i_{1}}, \quad x_{i_{2}}=\omega^{2} x_{i_{1}}, \quad \sigma_{1}\left(x_{0}, \ldots, x_{5}\right)=0 .
$$

The 40 planes $\Pi_{i_{1} i_{2} i_{3}}^{ \pm}$form one $\mathfrak{S}_{6}$-orbit. On the other hand, these 40 planes split into two $G$-orbits of length 20 , one containing the planes $\Pi_{i_{1} i_{2} i_{3}}^{ \pm}$for $0 \leqslant i_{1}<i_{2}<i_{3} \leqslant 4$, and the other containing $\Pi_{i_{1} i_{2} 5}^{ \pm}$for $0 \leqslant i_{1}<i_{2} \leqslant 4$.

Consider the following intersection form on the lattice $\mathrm{Cl}(X)$. Choose a general (smooth) hyperplane section $H$ of $X$; in particular, we assume that $H$ does not pass through the singular points of $X$. Given two Weil divisors $D_{1}$ and $D_{2}$ on $X$, we restrict them to $H$ (which makes sense since in appropriately chosen neighborhoods of the intersection of their supports with $H$ they are actually Cartier divisors), and define $D_{1} \bullet D_{2}$ as the intersection of the resulting curves on $H$.

Lemma 2.13. Choose two triples of indices

$$
0 \leqslant i_{1}<i_{2}<i_{3} \leqslant 5, \quad 0 \leqslant j_{1}<j_{2}<j_{3} \leqslant 5 .
$$

Let $c$ be the cardinality of the set $\left\{i_{1}, i_{2}, i_{3}\right\} \cap\left\{j_{1}, j_{2}, j_{3}\right\}$. If $c=2$, put $\delta=1$ provided that one can choose indices $1 \leqslant a<b \leqslant 3$ and $1 \leqslant a^{\prime}<b^{\prime} \leqslant 3$ such that $i_{a}=j_{a^{\prime}}, i_{b}=j_{b^{\prime}}$, and $b-a=b^{\prime}-a^{\prime}$; otherwise put $\delta=0$. The following assertions hold:

(o) if $c=0$, then $\Pi_{i_{1} i_{2} i_{3}}^{+} \bullet \Pi_{j_{1} j_{2} j_{3}}^{+}=\Pi_{i_{1} i_{2} i_{3}}^{+} \bullet \Pi_{j_{1} j_{2} j_{3}}^{-}=\Pi_{i_{1} i_{2} i_{3}}^{-} \bullet \Pi_{j_{1} j_{2} j_{3}}^{-}=1$;

(i) if $c=1$, then $\Pi_{i_{1} i_{2} i_{3}}^{+} \bullet \Pi_{j_{1} j_{2} j_{3}}^{+}=\Pi_{i_{1} i_{2} i_{3}}^{+} \bullet \Pi_{j_{1} j_{2} j_{3}}^{-}=\Pi_{i_{1} i_{2} i_{3}}^{-} \bullet \Pi_{j_{1} j_{2} j_{3}}^{-}=0$;

(ii) if $c=2$, then $\Pi_{i_{1} i_{2} i_{3}}^{+} \bullet \Pi_{j_{1} j_{2} j_{3}}^{+}=\Pi_{i_{1} i_{2} i_{3}}^{-} \bullet \Pi_{j_{1} j_{2} j_{3}}^{-}=\delta$, and $\Pi_{i_{1} i_{2} i_{3}}^{+} \bullet \Pi_{j_{1} j_{2} j_{3}}^{-}=1-\delta$;

(iii) one has $\Pi_{i_{1} i_{2} i_{3}}^{+} \bullet \Pi_{i_{1} i_{2} i_{3}}^{+}=\Pi_{i_{1} i_{2} i_{3}}^{-} \bullet \Pi_{i_{1} i_{2} i_{3}}^{-}=-2$, and $\Pi_{i_{1} i_{2} i_{3}}^{+} \bullet \Pi_{i_{1} i_{2} i_{3}}^{-}=1$.

Proof. The self-intersection number -2 corresponds to the self-intersection of a smooth rational curve on a $K 3$ surface. The cases with intersection number 1 correspond to pairs of planes that meet along a line, and the cases with intersection number 0 correspond to pairs of planes that meet at a point.

Corollary 2.14. The $20 \times 20$ matrix of intersection numbers of the planes $\Pi_{i_{1} i_{2} i_{3}}^{ \pm}$, where $0 \leqslant i_{1}<i_{2}<i_{3} \leqslant 4$, has rank 16 . Similarly, the $20 \times 20$ matrix of intersection numbers of the planes $\Pi_{i_{1} i_{2} 5}^{ \pm}$, where $0 \leqslant i_{1}<i_{2} \leqslant 4$, also has rank 16 .

Proof. Straightforward computation.

Lemma 2.4 and Corollary 2.14 imply the following result.

Corollary 2.15. The classes of the 20 planes $\Pi_{i_{1} i_{2} i_{3}}^{ \pm}$, where $0 \leqslant i_{1}<i_{2}<i_{3} \leqslant 4$, generate the $\mathbb{Q}$-vector space

$$
\mathrm{Cl}(X)_{\mathbb{Q}}=\mathrm{Cl}(X) \otimes \mathbb{Q} .
$$

Similarly, the classes of the 20 planes $\Pi_{i_{1} i_{2} 5}^{ \pm}$, where $0 \leqslant i_{1}<i_{2} \leqslant 4$, also generate the $\mathbb{Q}$-vector space $\mathrm{Cl}(X)_{\mathbb{Q}}$.

By Corollary 2.8, the assertion of Proposition 2.9 follows from Corollary 2.15, This completes the proof of Theorem 1.1. 


\section{BARTH SEXTIC DOUBLE SOLID}

Let $\mathbb{I}$ be the trivial representation of the group $\mathfrak{A}_{5}$, and let $\mathbb{V}$ be one of its two three-dimensional irreducible representations (see e.g. $\left[\mathrm{C}^{+}\right.$, p. 2]). Put $\mathbb{P}^{3}=\mathbb{P}(\mathbb{I} \oplus \mathbb{V})$. By Ba96, there exists a unique $\mathfrak{A}_{5}$-invariant sextic surface $\mathcal{B}$ in $\mathbb{P}^{3}$ with 65 isolated singular points. Moreover, the surface $\mathcal{B}$ is given in appropriate homogeneous coordinates $x_{0}, x_{1}, x_{2}, x_{3}$ by equation

$$
4\left(\tau^{2} x_{0}^{2}-x_{1}^{2}\right)\left(\tau^{2} x_{1}^{2}-x_{2}^{2}\right)\left(\tau^{2} x_{2}^{2}-x_{0}^{2}\right)-(1+2 \tau) x_{3}^{2}\left(x_{0}^{2}+x_{1}^{2}+x_{2}^{2}-x_{3}^{2}\right)^{2}=0,
$$

where $\tau=\frac{1+\sqrt{5}}{2}$, and it has only nodes as singularities.

Recall from [Ba96, §1] that the group $\mathfrak{A}_{5}$ acting on $\mathbb{P}^{3}$ so that the sextic $\mathcal{B}$ is $\mathfrak{A}_{5}$-invariant can be thought of as the group of rotations of an icosahedron with 12 vertices

$$
[ \pm \tau: \pm 1: 0: 1], \quad[0: \pm \tau: \pm 1: 1], \quad[ \pm 1: 0: \pm \tau: 1]
$$

In particular, the group $\mathfrak{A}_{5}$ contains the transformation

$$
x_{0} \mapsto-x_{0}, \quad x_{1} \mapsto-x_{1}, \quad x_{2} \mapsto x_{2}, \quad x_{3} \mapsto x_{3}
$$

of order 2 , the transformation

$$
x_{0} \mapsto x_{1} \mapsto x_{2} \mapsto x_{0}, \quad x_{3} \mapsto x_{3}
$$

of order 3 , and the transformation

$$
\left(x_{0}, x_{1}, x_{2}\right) \mapsto\left(x_{0}, x_{1}, x_{2}\right) \mathrm{M}^{T}, \quad x_{3} \mapsto x_{3},
$$

where $\mathrm{M}$ is the matrix

$$
\begin{aligned}
\left(\begin{array}{ccc}
\frac{\tau}{\sqrt{\tau+2}} & -\frac{1}{\sqrt{\tau+2}} & 0 \\
\frac{1}{\sqrt{\tau+2}} & \frac{\tau}{\sqrt{\tau+2}} & 0 \\
0 & 0 & 1
\end{array}\right) & \left(\begin{array}{ccc}
1 & 0 & 0 \\
0 & \cos \left(\frac{2 \pi}{5}\right) & -\sin \left(\frac{2 \pi}{5}\right) \\
0 & \sin \left(\frac{2 \pi}{5}\right) & \cos \left(\frac{2 \pi}{5}\right)
\end{array}\right)\left(\begin{array}{ccc}
\frac{\tau}{\sqrt{\tau+2}} & -\frac{1}{\sqrt{\tau+2}} & 0 \\
\frac{1}{\sqrt{\tau+2}} & \frac{\tau}{\sqrt{\tau+2}} & 0 \\
0 & 0 & 1
\end{array}\right)^{-1}= \\
& =\left(\begin{array}{ccc}
\frac{\sqrt{5}(3+\sqrt{5})}{2(5+\sqrt{5})} & \frac{\sqrt{5}}{5+\sqrt{5}} & \frac{1}{2} \\
\frac{\sqrt{5}}{5+\sqrt{5}} & \frac{1}{2} & \frac{-\sqrt{5}-1}{4} \\
-\frac{1}{2} & \frac{\sqrt{5}+1}{4} & \frac{\sqrt{5}-1}{4}
\end{array}\right)=\frac{1}{2}\left(\begin{array}{ccc}
\tau & \tau-1 & 1 \\
\tau-1 & 1 & -\tau \\
-1 & \tau & \tau-1
\end{array}\right) .
\end{aligned}
$$

The latter is a transformation of order 5 which corresponds to a rotation around the axis through the vertices $[\tau: 1: 0: 1]$ and $[-\tau:-1: 0: 1]$ of the icosahedron by the angle $2 \pi / 5$.

Let $\Sigma_{15}$ be the $\mathfrak{A}_{5}$-orbit of the point $[1: 0: 0: 0]$, let $\Sigma_{30}$ be the $\mathfrak{A}_{5}$-orbit of the point $[1: 0: 0: 1]$, and let $\Sigma_{20}$ be the $\mathfrak{A}_{5}$-orbit of the point $[1: 1: 1: 1]$. Then one has $\left|\Sigma_{k}\right|=k$, and one can check that the sextic surface $\mathcal{B}$ is singular at the points of these three $\mathfrak{A}_{5}$-orbits. Moreover, one has $\operatorname{Sing}(\mathcal{B})=\Sigma_{15} \cup \Sigma_{20} \cup \Sigma_{30}$, see [Ba96, §1].

Remark 3.1. Restricting the left hand side of (3.1) to the plane $x_{3}=x_{0}+x_{1}+x_{2}$ we get an equation

$$
-4(5 \tau+3)\left((\tau-2)\left(x_{0} x_{1}^{2}+x_{1} x_{2}^{2}+x_{2} x_{0}^{2}\right)+(\tau-3) x_{0} x_{1} x_{2}-\left(x_{0}^{2} x_{1}+x_{1}^{2} x_{2}+x_{2}^{2} x_{0}\right)\right)^{2}=0 .
$$

Similarly, restricting the left hand side of (3.1) to the plane $x_{3}=x_{0}-x_{1}-x_{2}$ we get an equation

$$
-4(5 \tau+3)\left((2-\tau)\left(x_{0} x_{1}^{2}-x_{1} x_{2}^{2}-x_{2} x_{0}^{2}\right)+(3-\tau) x_{0} x_{1} x_{2}-\left(x_{0}^{2} x_{1}+x_{1}^{2} x_{2}-x_{2}^{2} x_{0}\right)\right)^{2}=0 .
$$


Define the plane $\Xi_{\left(v_{0}, v_{1}, v_{2}\right)}$ in $\mathbb{P}^{3}$ by equation $x_{3}=v_{0} x_{0}+v_{1} x_{1}+v_{2} x_{2}$, where $v=\left(v_{0}, v_{1}, v_{2}\right)$ is one of the following collections of coefficients:

$$
\begin{aligned}
& (1,1,1),(1,1,-1),(1,-1,1),(-1,1,1), \\
& (1,-1,-1),(-1,1,-1),(-1,-1,1),(-1,-1,-1), \\
& (\tau-1, \tau, 0),(1-\tau, \tau, 0),(1-\tau,-\tau, 0),(\tau-1,-\tau, 0),(\tau, 0,1-\tau),(\tau, 0, \tau-1), \\
& (-\tau, 0, \tau-1),(-\tau, 0,1-\tau),(0, \tau-1, \tau),(0, \tau-1,-\tau),(0,1-\tau,-\tau),(0,1-\tau, \tau) .
\end{aligned}
$$

There are 20 planes like this, and they form a single $\mathfrak{A}_{5}$-orbit. Similarly, define the plane $\Theta_{\left(u_{0}, u_{1}, u_{2}\right)}$ in $\mathbb{P}^{3}$ by equation $u_{0} x_{0}+u_{1} x_{1}+u_{2} x_{2}=0$, where $u=\left(u_{0}, u_{1}, u_{2}\right)$ is one of the following collections of coefficients:

$$
(\tau, 1,0),(\tau,-1,0),(0, \tau, 1),(0, \tau,-1),(1,0, \tau),(-1,0, \tau) .
$$

There are 6 planes like this, and they form a single $\mathfrak{A}_{5}$-orbit.

Lemma 3.2. A restriction of the sextic $\mathcal{B}$ to each of the planes $\Xi_{v}$ is a smooth cubic curve taken with multiplicity 2. A restriction of the sextic $\mathcal{B}$ to each of the planes $\Theta_{u}$ is a union of a line taken with multiplicity 2 and an irreducible conic taken with multiplicity 2.

Proof. It is enough to check the assertion for one of the planes $\Xi_{v}$ and one of the planes $\Theta_{u}$. The restriction of $\mathcal{B}$ to the plane $\Xi_{1,1,1}$ is given by equation

$$
\left((\tau-2)\left(x_{0} x_{1}^{2}+x_{1} x_{2}^{2}+x_{2} x_{0}^{2}\right)+(\tau-3) x_{0} x_{1} x_{2}-\left(x_{0}^{2} x_{1}+x_{1}^{2} x_{2}+x_{2}^{2} x_{0}\right)\right)^{2}=0,
$$

see Remark 3.1. Similarly, the restriction of $\mathcal{B}$ to the plane $\Theta_{(-1,0, \tau)}$ is given by equation

$$
x_{3}^{2}\left(x_{1}^{2}+\left(1+\tau^{2}\right) x_{2}^{2}-x_{3}^{2}\right)^{2}=0 .
$$

Denote by $\Upsilon$ the plane in $\mathbb{P}^{3}$ that is given by $x_{3}=0$, so that $\Upsilon \cong \mathbb{P}(\mathbb{V})$. Recall that for every $k \in\{6,10,15\}$ there is a unique $\mathfrak{A}_{5}$-orbit $\Omega_{k}$ of length $k$ in $\Upsilon$, and there is a unique $\mathfrak{A}_{5}$-invariant curve $\mathcal{L}_{k}$ in $\Upsilon$ that is a union of $k$ lines, see e.g. [CS15, Lemma 5.3.1(i),(ii)].

Lemma 3.3. Let $\ell$ be a line in $\Upsilon$ that is not an irreducible component of $\mathcal{L}_{6}$. Suppose that $\left(\ell \cdot \mathcal{L}_{6}\right)_{P} \geqslant 2$ for every point $P \in \ell \cap \mathcal{L}_{6}$. Then $\ell$ is an irreducible component of $\mathcal{L}_{10}$.

Proof. By [CS15, Theorem 6.1.2(i)] the singular points of the curve $\mathcal{L}_{6}$ are the points of $\Omega_{15}$; the multiplicity of $\mathcal{L}_{6}$ at each of these points equals 2 . Therefore, the line $\ell$ must contain three points of $\Omega_{15}$. On the other hand, all lines passing through pairs of points of $\Omega_{15}$ are irreducible components of the curves $\mathcal{L}_{6}$, or $\mathcal{L}_{10}$, or $\mathcal{L}_{15}$; this follows from polarity (see [CS15, Remark 5.3.2]) and the fact that the points of pairwise intersections of the irreducible components of $\mathcal{L}_{15}$ are

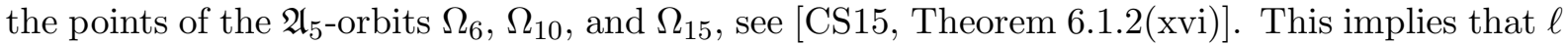
is an irreducible component of either $\mathcal{L}_{6}$, or $\mathcal{L}_{10}$, or $\mathcal{L}_{15}$. However, the first of these cases does not occur by assumption, and the third is excluded by [CS15, Theorem 6.1.2(xiv)].

Note that the intersection $\Xi_{v} \cap \Upsilon$ is an irreducible component of $\mathcal{L}_{10}$. Indeed, this intersection is a line in $\Upsilon$ whose $\mathfrak{A}_{5}$-orbit has length $k$ that divides 20 , and moreover $k<20$ because $\Xi_{(1,1,1)}$ and $\Xi_{(-1,-1,-1)}$ intersect $\Upsilon$ by the same line. Similarly, the intersection $\Theta_{u} \cap \Upsilon$ is an irreducible component of $\mathcal{L}_{6}$, because the latter is the only $\mathfrak{A}_{5}$-orbit in $\Upsilon$ that consists of at most 6 lines.

Proposition 3.4. Suppose that $\Pi$ is a plane in $\mathbb{P}^{3}$ such that the restriction $\left.\mathcal{B}\right|_{\Pi}$ is a cubic curve taken with multiplicity 2 . Then $\Pi$ is one of the planes $\Xi_{v}$ or $\Theta_{u}$. 
Proof. Observe that $\Pi \neq \Upsilon$, because $\left.\mathcal{B}\right|_{\Upsilon}=\mathcal{L}_{6}$ is a reduced curve. So we put $\ell=\Pi \cap \Upsilon$. By Lemma 3.3, the line $\ell$ is an irreducible component of either $\mathcal{L}_{6}$ or $\mathcal{L}_{10}$. Since $\mathfrak{A}_{5}$ permutes transitively the irreducible components of each of the curves $\mathcal{L}_{6}$ and $\mathcal{L}_{10}$, we may assume that $\ell$ is given either by $\tau x_{0}+x_{1}=x_{3}=0$, or by $x_{0}+x_{1}+x_{2}=x_{3}=0$.

Suppose that $\ell$ is given by $\tau x_{0}+x_{1}=x_{3}=0$. Then $\Pi$ is given by the equation $\tau x_{0}+x_{1}=\lambda x_{3}$ for some $\lambda \in \mathbb{C}$. If $\lambda=0$, then $\Pi=\Theta_{(\tau, 1,0)}$. If $\lambda \neq 0$, then the restriction $\left.\mathcal{B}\right|_{\Pi}$ is given by

$$
\begin{aligned}
0=4\left(\tau^{2} x_{0}^{2}-x_{1}^{2}\right)\left(\tau^{2} x_{1}^{2}-x_{2}^{2}\right)\left(\tau^{2} x_{2}^{2}\right. & \left.-x_{0}^{2}\right) \\
& -\frac{1+2 \tau}{\lambda^{2}}\left(\tau x_{0}+x_{1}\right)^{2}\left(x_{0}^{2}+x_{1}^{2}+x_{2}^{2}-\frac{1}{\lambda^{2}}\left(\tau x_{0}+x_{1}\right)^{2}\right)^{2} .
\end{aligned}
$$

This (possibly non-reduced) sextic curve contains the line $\tau x_{0}+x_{1}=0$ with multiplicity 1 , so that it cannot be a double cubic.

Now we suppose that $\ell$ is given by $x_{0}+x_{1}+x_{2}=x_{3}=0$. Then the plane $\Pi$ is given by the equation $x_{0}+x_{1}+x_{2}=\lambda x_{3}$ for some $\lambda \in \mathbb{C}$. If $\lambda=1$, then $\Pi=\Xi_{(1,1,1)}$. If $\lambda=-1$, then $\Pi=\Xi_{(-1,-1,-1)}$.

Suppose that $\lambda \neq 0$. Put $\mu=\frac{1}{\lambda}$. Then $\mu \neq 0$, and the restriction $\left.\mathcal{B}\right|_{\Pi}$ is given by the equation $f\left(x_{0}, x_{1}, x_{2}\right)=0$, where

$$
\begin{aligned}
f\left(x_{0}, x_{1}, x_{2}\right)=4\left(\tau^{2} x_{0}^{2}-x_{1}^{2}\right) & \left(\tau^{2} x_{1}^{2}-x_{2}^{2}\right)\left(\tau^{2} x_{2}^{2}-x_{0}^{2}\right) \\
& -(1+2 \tau) \mu^{2}\left(x_{0}+x_{1}+x_{2}\right)^{2}\left(x_{0}^{2}+x_{1}^{2}+x_{2}^{2}-\mu^{2}\left(x_{0}+x_{1}+x_{2}\right)^{2}\right)^{2} .
\end{aligned}
$$

We have to show that the polynomial $f\left(x_{0}, x_{1}, x_{2}\right)$ is not a square of a cubic polynomial unless $\mu= \pm 1$. To show this it is enough to prove the same assertion for the polynomial

$$
\begin{aligned}
f\left(1, x_{1},-x_{1}\right)=-(8 \tau+4) x_{1}^{6} & -(4+8 \tau)\left(\mu^{2}-3\right) x_{1}^{4} \\
& +(4+8 \tau)\left(\mu^{2}-\tau\right)\left(\mu^{2}+\tau-1\right) x_{1}^{2}-(1+2 \tau) \mu^{2}(\mu+1)^{2}(\mu-1)^{2} .
\end{aligned}
$$

This follows from the fact that if $\mu \neq \pm 1$, then both the constant term and the leading coefficient of $f\left(1, x_{1},-x_{1}\right)$ are not zero, while the coefficients at $x_{1}$ and $x_{1}^{3}$ are both zero.

Thus, we see that $\lambda=0$, so that $\Pi$ is given by $x_{0}+x_{1}+x_{2}=0$. Expressing $x_{0}=-x_{1}-x_{2}$, we see that the restriction $\left.\mathcal{B}\right|_{\Pi}$ is given by the equation $g\left(x_{1}, x_{2}, x_{3}\right)=0$, where

$$
\begin{aligned}
g\left(x_{1}, x_{2}, x_{4}\right)=4\left(\tau^{2}\left(x_{1}+x_{2}\right)^{2}-x_{1}^{2}\right)\left(\tau^{2} x_{1}^{2}-x_{2}^{2}\right) & \left(\tau^{2} x_{2}^{2}-\left(x_{1}+x_{2}\right)^{2}\right) \\
& -(1+2 \tau) x_{3}^{2}\left(\left(x_{1}+x_{2}\right)^{2}+x_{1}^{2}+x_{2}^{2}-x_{3}^{2}\right)^{2} .
\end{aligned}
$$

We have to show that the polynomial $g\left(x_{1}, x_{2}, x_{3}\right)$ is not a square of a cubic polynomial. To show this it is enough to prove the same assertion for the polynomial

$$
g\left(x_{1},-x_{1}, 1\right)=-(1+2 \tau)\left(4 x_{1}^{6}+4 x_{1}^{4}-4 x_{1}^{2}+1\right) .
$$

This polynomial is not a square of a cubic polynomial, because both its constant term and the leading coefficient are not zero, while the coefficients at $x_{1}$ and $x_{1}^{3}$ are both zero.

Let $\pi: X \rightarrow \mathbb{P}^{3}$ be a double cover branched over the sextic $\mathcal{B}$. The equation of $X$ can be written in the weighted projective space $\mathbb{P}(1,1,1,1,2)$ with weighted homogeneous coordinates $x_{0}, \ldots, x_{3}$, and $w$ as

$$
w^{2}+4 l_{1} l_{2} l_{3} l_{4} l_{5} l_{6}-q_{3}^{2}=0
$$

where $l_{1}=\tau x_{0}-x_{1}, l_{2}=\tau x_{1}-x_{2}, l_{3}=\tau x_{2}-x_{0}, l_{4}=\tau x_{0}+x_{1}, l_{5}=\tau x_{1}+x_{2}, l_{6}=\tau x_{2}+x_{0}$, and

$$
q_{3}=\sqrt{1+2 \tau} x_{3}\left(x_{0}^{2}+x_{1}^{2}+x_{2}^{2}-x_{3}^{2}\right) .
$$


The class group of the threefold $X$ was described by Endrass.

Lemma 3.5 ([En99, Example 3.7]). One has $\operatorname{rk} \mathrm{Cl}(X)=14$.

Proposition 3.6. The threefold $X$ is rational.

Proof. Making a change of coordinates $w=2 y l_{1} l_{2}+q_{3}$ we see that there is a birational map $\varphi: X \rightarrow Y$ to a quartic threefold $Y$ given in the projective space $\mathbb{P}^{4}$ with homogeneous coordinates $x_{0}, \ldots, x_{3}, y$ by equation $y^{2} l_{1} l_{2}+y q_{3}+l_{3} l_{4} l_{5} l_{6}=0$. The map $\varphi$ is given by the formula

$$
\left[x_{0}: x_{1}: x_{2}: x_{3}: w\right] \mapsto\left[2 l_{1} l_{2} x_{0}: 2 l_{1} l_{2} x_{1}: 2 l_{1} l_{2} x_{2}: 2 l_{1} l_{2} x_{3}: w-q_{3}\right] .
$$

The inverse birational map $\psi: Y \rightarrow X$ is given by the Stein factorization of the linear projection from the point $[0: 0: 0: 0: 1]$ in $\mathbb{P}^{4}$, so that $\psi$ is defined by the formula

$$
\left[x_{0}: x_{1}: x_{2}: x_{3}: y\right] \mapsto\left[x_{0}: x_{1}: x_{2}: x_{3}: 2 l_{1} l_{2} y+q_{3}\right] .
$$

The quartic $Y$ contains a plane $\Pi$ given by equations $y=l_{4}=0$. The projection $\sigma: Y \rightarrow \rightarrow \mathbb{P}^{1}$ from $\Pi$ is given by $[\lambda: \mu]=\left[y: l_{4}\right]$, where $\lambda$ and $\mu$ are homogeneous coordinates on $\mathbb{P}^{1}$.

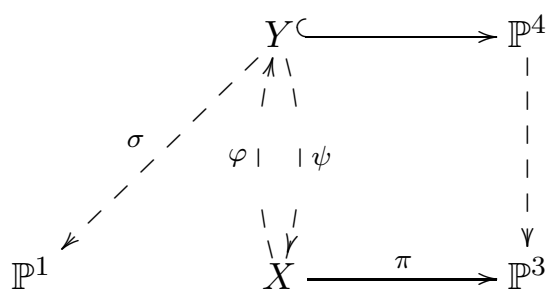

Putting $\lambda=\frac{y}{l_{4}}$ and $\mu=1$, we see that the general fiber of $\sigma$ (in a scheme sence) is a cubic surface in the projective space $\mathbb{P}_{\mathbb{F}}^{3}$ over the field $\mathbb{F}=\mathbb{C}(\lambda)$, that is given by equation

$$
\lambda^{2} l_{1} l_{2} l_{4}+\lambda q_{3}+l_{3} l_{5} l_{6}=0 .
$$

Here we use $x_{0}, \ldots, x_{3}$ also as a homogeneous coordinates on $\mathbb{P}_{\mathbb{F}}^{3}$.

Restricting the left hand side of equation (3.7) to the plane $\Pi^{\prime}$ given by $x_{3}=x_{0}+x_{1}+x_{2}$ and using Remark 3.1, we see that the corresponding curve is given by equation

$$
\left(\lambda l_{4}+\sqrt{2 \tau+1}(2 \tau-3) l_{3}\right)\left(\lambda l_{1} l_{2}+\sqrt{2 \tau+1} l_{5} l_{6}\right)=0 .
$$

Restricting the left hand side of equation (3.7) to the plane $\Pi^{\prime \prime}$ given by $x_{3}=x_{0}-x_{1}-x_{2}$ and using Remark 3.1, we see that the corresponding curve is given by equation

$$
\left(\lambda l_{1}+\sqrt{2 \tau+1}(2 \tau-3) l_{6}\right)\left(\lambda l_{2} l_{4}+\sqrt{2 \tau+1} l_{3} l_{5}\right)=0 .
$$

One can check that the lines in $\mathbb{P}_{\mathbb{F}}^{3}$ given by equations

$$
x_{3}-x_{0}-x_{1}-x_{2}=\lambda l_{4}+\sqrt{2 \tau+1}(2 \tau-3) l_{3}=0
$$

and

$$
x_{3}-x_{0}+x_{1}+x_{2}=\lambda l_{1}+\sqrt{2 \tau+1}(2 \tau-3) l_{6}=0
$$

are disjoint. Since they are contained in the cubic surface (3.7), we see that the cubic surface (3.7) is rational over the field $\mathbb{F}$, so that both $Y$ and $X$ are rational (over the field $\mathbb{C}$ ).

Now we are going to describe the generators of the group $\mathrm{Cl}(X)$.

The intersection of $X$ with the hypersurface $x_{3}=x_{0}+x_{1}+x_{2}$ splits as a union of two surfaces $\Xi_{(1,1,1)}^{+}$and $\Xi_{(1,1,1)}^{-}$that are given by

$$
\left\{\begin{array}{l}
x_{3}=x_{0}+x_{1}+x_{2}, \\
w=C_{ \pm} \cdot\left((\tau-2)\left(x_{0} x_{1}^{2}+x_{1} x_{2}^{2}+x_{2} x_{0}^{2}\right)+(\tau-3) x_{0} x_{1} x_{2}-\left(x_{0}^{2} x_{1}+x_{1}^{2} x_{2}+x_{2}^{2} x_{0}\right)\right),
\end{array}\right.
$$


respectively, where $C_{ \pm}= \pm 2 \sqrt{5 \tau+3}$. The image $\pi\left(\Xi_{(1,1,1)}^{+}\right)=\pi\left(\Xi_{(1,1,1)}^{-}\right)$is the plane in $\mathbb{P}^{3}$ that is given by equation $x_{3}=x_{0}+x_{1}+x_{2}$, cf. Lemma 3.2. Since the $\mathfrak{A}_{5}$-orbit of the

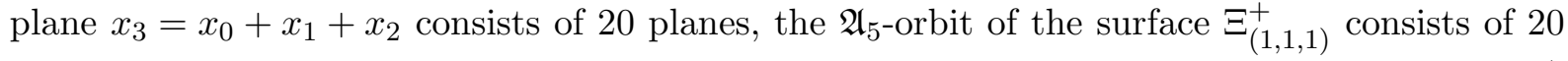
surfaces. Similarly, the $\mathfrak{A}_{5}$-orbit of the surface $\Xi_{(1,1,1)}^{-}$also consists of 20 surfaces. Denote by $\Xi_{v}^{+}$ the surface in the the $\mathfrak{A}_{5}$-orbit of $\Xi_{(1,1,1)}^{+}$such that $\pi\left(\Xi_{v}^{+}\right)=\Xi_{v}$, where $v=\left(v_{0}, v_{1}, v_{2}\right)$ is one of the collections of coefficients listed in (3.5).

Remark 3.7. The anticanonical degree of the surfaces $\Xi_{v}^{ \pm}$equals 1 , i.e. one has $\Xi_{v}^{ \pm} \cdot K_{X}^{2}=1$. By Lemma 3.2, the preimage on $X$ of a plane $\Theta_{u}$ also splits as a union of two surfaces $\Theta_{u}^{+}$ and $\Theta_{u}^{-}$of anticanonical degree 1. It follows from Proposition 3.4 that there are no surfaces of anticanonical degree 1 on $X$ except $\Xi_{v}^{ \pm}$and $\Theta_{u}^{ \pm}$.

Fix a sufficiently general (smooth) $K 3$ surface $S$ in the linear system $\left|-K_{X}\right|$. For every two surfaces $\Xi_{v}^{+}$and $\Xi_{v^{\prime}}^{+}$, put

$$
\Xi_{v}^{+} \bullet \Xi_{v^{\prime}}^{+}=\left.\left.\Xi_{v}^{+}\right|_{S} \cdot \Xi_{v^{\prime}}^{+}\right|_{S}
$$

Then $\Xi_{v}^{+} \bullet \Xi_{v}^{+}=-2$ by the adjunction formula. Moreover, if $v \neq v^{\prime}$, then either $\Xi_{v}^{+} \bullet \Xi_{v^{\prime}}^{+}=1$ or $\Xi_{v}^{+} \bullet \Xi_{v^{\prime}}^{+}=0$ by construction. Furthermore, if $v \neq v^{\prime}$, then $\Xi_{v}^{+} \bullet \Xi_{v^{\prime}}^{+}=0$ if and only if the intersection $\Xi_{v}^{+} \cap \Xi_{v^{\prime}}^{+}$consists of finitely many points.

Denote by $N, R$, and $M$ the transformations (3.2), (3.3), and (3.4), respectively.

Example 3.8. By Remark 3.1, the surface $\Xi_{(1,1,1)}^{+}$is defined in $\mathbb{P}(1,1,1,1,2)$ by equations

$$
\left\{\begin{array}{l}
x_{3}=x_{0}+x_{1}+x_{2} \\
w=(\tau-2)\left(x_{0} x_{1}^{2}+x_{1} x_{2}^{2}+x_{2} x_{0}^{2}\right)+(\tau-3) x_{0} x_{1} x_{2}-\left(x_{0}^{2} x_{1}+x_{1}^{2} x_{2}+x_{2}^{2} x_{0}\right),
\end{array}\right.
$$

The transformation $M^{3}$ is given by the matrix

$$
\mathrm{M}^{3}=\frac{1}{2}\left(\begin{array}{ccc}
1 & \tau & 1-\tau \\
\tau & 1-\tau & 1 \\
\tau-1 & -1 & -\tau
\end{array}\right)
$$

Thus the surface $\Xi_{(1,1,-1)}^{+}$is defined by equations

$$
\left\{\begin{array}{l}
x_{3}=x_{0}+x_{1}-x_{2} \\
w=(\tau-2)\left(x_{0} x_{1}^{2}+x_{1} x_{2}^{2}-x_{2} x_{0}^{2}\right)-(\tau-3) x_{0} x_{1} x_{2}-\left(x_{0}^{2} x_{1}-x_{1}^{2} x_{2}+x_{2}^{2} x_{0}\right) .
\end{array}\right.
$$

Therefore, the intersection $\Xi_{(1,1,1)}^{+} \cap \Xi_{(1,-1,-1)}^{+}$is the line in $\Xi_{(1,1,1)}^{+}$that is cut out by an equation $x_{2}=0$, so that $\Xi_{(1,1,1)}^{+} \bullet \Xi_{(1,-1,-1)}^{+}=1$.

Example 3.9. The transformation $R N$ is given by the matrix

$$
\mathrm{RN}=\left(\begin{array}{ccc}
0 & 0 & 1 \\
-1 & 0 & 0 \\
0 & -1 & 0
\end{array}\right)
$$

Thus the surface $\Xi_{(1,-1,-1)}^{+}$is defined in $\mathbb{P}(1,1,1,1,2)$ by equations

$$
\left\{\begin{array}{l}
x_{3}=x_{0}-x_{1}-x_{2}, \\
w=(\tau-2)\left(x_{0} x_{1}^{2}-x_{1} x_{2}^{2}-x_{2} x_{0}^{2}\right)+(\tau-3) x_{0} x_{1} x_{2}+\left(x_{0}^{2} x_{1}+x_{1}^{2} x_{2}-x_{2}^{2} x_{0}\right) .
\end{array}\right.
$$

Therefore, the intersection $\Xi_{(1,1,1)}^{+} \cap \Xi_{(1,-1,-1)}^{+}$is defined by equations

$$
\left\{\begin{array}{l}
x_{1}+x_{2}=0 \\
(\tau-2)\left(x_{1} x_{2}^{2}+x_{2} x_{0}^{2}\right)=x_{0}^{2} x_{1}+x_{1}^{2} x_{2}
\end{array}\right.
$$


This system of equations defines a set consisting of three points, and $\Xi_{(1,1,1)}^{+} \bullet \Xi_{(1,-1,-1)}^{+}=0$.

In the similar way one gets $\Xi_{v}^{+}=T_{v}\left(\Xi_{(1,1,1)}^{+}\right)$, where $T_{v}$ is an element in $\mathfrak{A}_{5}$ given by Table 1 .

TABlE 1. The surfaces $\Xi_{v}^{+}$

\begin{tabular}{|c|c|c|c|c|c|}
\hline$v$ & $(1,1,1)$ & $(1,1,-1)$ & $(1,-1,1)$ & $(-1,1,1)$ & $(1,-1,-1)$ \\
\hline$T_{v}$ & $\mathrm{Id}$ & $M^{3}$ & $R^{2} M^{3}$ & $R M^{3}$ & $R N$ \\
\hline \hline$v$ & $(-1,1,-1)$ & $(-1,-1,1)$ & $(-1,-1,-1)$ & $(\tau-1, \tau, 0)$ & $(1-\tau, \tau, 0)$ \\
\hline$T_{v}$ & $R^{2} N$ & $N$ & $M^{2} N$ & $M^{4}$ & $R M^{2}$ \\
\hline \hline$v$ & $(1-\tau,-\tau, 0)$ & $(\tau-1,-\tau, 0)$ & $(\tau, 0,1-\tau)$ & $(\tau, 0, \tau-1)$ & $(-\tau, 0, \tau-1)$ \\
\hline$T_{v}$ & $M N$ & $R M^{4} N$ & $M^{2}$ & $M$ & $M^{4} N$ \\
\hline \hline$v$ & $(-\tau, 0,1-\tau)$ & $(0, \tau-1, \tau)$ & $(0, \tau-1,-\tau)$ & $(0,1-\tau,-\tau)$ & $(0,1-\tau, \tau)$ \\
\hline$T_{v}$ & $M^{3} N$ & $R M^{4}$ & $R^{2} M^{4} N$ & $R M N$ & $R^{2} M^{2}$ \\
\hline
\end{tabular}

This leads to the following result.

Lemma 3.10. One has

$$
\Xi_{(1,1,1)}^{+} \bullet \Xi_{v}^{+}=\left\{\begin{aligned}
0 \text { if } v= & (1,-1,-1),(-1,1,-1),(-1,-1,1),(-1,-1,-1), \\
& (\tau-1,-\tau, 0),(-\tau, 0, \tau-1),(0, \tau-1,-\tau), \\
1 \text { if } v= & (1,1,-1),(1,-1,1),(-1,1,1),(\tau-1, \tau, 0),(1-\tau, \tau, 0), \\
& (1-\tau,-\tau, 0),(\tau, 0,1-\tau),(\tau, 0, \tau-1),(-\tau, 0,1-\tau), \\
& (0, \tau-1, \tau),(0,1-\tau,-\tau),(0,1-\tau, \tau), \\
-2 \text { if } v= & (1,1,1) .
\end{aligned}\right.
$$

Similarly, we can compute all possible values of $\Xi_{v}^{+} \bullet \Xi_{v^{\prime}}^{+}$. They are given in Table 2 below. The intersection matrix for the surfaces $\Xi_{v}^{-}$is the same as one given by Table 2 ,

Corollary 3.11. The $20 \times 20$ matrix of intersection numbers of the surfaces $\Xi_{v}^{+}$, where the index $v$ is taken from the list (3.5), has rank 14. Similarly, the $20 \times 20$ matrix of intersection numbers of the surfaces $\Xi_{v}^{-}$has rank 14 .

Proof. Straightforward computation.

Lemma 3.5 and Corollary 3.11 imply the following result.

Corollary 3.12. The classes of the 20 surfaces $\Xi_{v}^{+}$, where the index $v$ is taken from the list (3.5), generate the $\mathbb{Q}$-vector space $\mathrm{Cl}(X)_{\mathbb{Q}}=\mathrm{Cl}(X) \otimes \mathbb{Q}$. Similarly, the classes of the 20 surfaces $\Xi_{v}^{-}$ also generate the $\mathbb{Q}$-vector space $\mathrm{Cl}(X)_{\mathbb{Q}}$.

Corollaries 2.8 and 3.12 imply

Proposition 3.13. The group $\mathrm{Cl}(X)^{G}$ is generated by $-K_{X}$.

Now we derive Theorem 1.2 from Proposition 3.13 .

Proof of Theorem 1.2. Since $\mathrm{Cl}(X)^{G}$ is generated by $-K_{X}$ by Proposition 3.13, the required assertion immediately follows from the proof of [CP10, Theorem A]. The only difference is that one should use a $G$-equivariant version of the standard Noether-Fano inequality, which is [CS15, Corollary 3.3.3]. 
TABLE 2. Intersection matrix for $\Xi_{v}^{+}$

\begin{tabular}{|c|c|c|c|c|c|c|c|c|c|c|c|c|c|c|c|c|c|c|c|c|}
\hline & $(1,1,1)$ & $(1,1,-1)$ & $(1,-1,1)$ & $(-1,1,1)$ & $(1,-1,-1)$ & $(-1,1,-1)$ & $(-1,-1,1)$ & $(-1,-1,-1)$ & $(\tau-1, \tau, 0)$ & $(1-\tau, \tau, 0)$ & $(1-\tau,-\tau, 0)$ & $(\tau-1,-\tau, 0)$ & $(\tau, 0,1-\tau)$ & $(\tau, 0, \tau-1)$ & $(-\tau, 0, \tau-1)$ & $(-\tau, 0,1-\tau)$ & $(0, \tau-1, \tau)$ & $(0, \tau-1,-\tau)$ & $(0,1-\tau,-\tau)$ & $(0,1-\tau, \tau)$ \\
\hline$(1,1,1)$ & -2 & 1 & 1 & 1 & 0 & 0 & 0 & 0 & 1 & 1 & 1 & 0 & 1 & 1 & 0 & 1 & 1 & 0 & 1 & 1 \\
\hline$(1,1,-1)$ & 1 & -2 & 0 & 0 & 1 & 1 & 0 & 0 & 1 & 1 & 1 & 0 & 1 & 1 & 1 & 0 & 0 & 1 & 1 & 1 \\
\hline$(1,-1,1)$ & 1 & 0 & -2 & 0 & 1 & 0 & 1 & 0 & 0 & 1 & 1 & 1 & 1 & 1 & 0 & 1 & 1 & 1 & 0 & 1 \\
\hline$(-1,1,1)$ & 1 & 0 & 0 & -2 & 0 & 1 & 1 & 0 & 1 & 1 & 0 & 1 & 1 & 0 & 1 & 1 & 1 & 0 & 1 & 1 \\
\hline$(1,-1,-1)$ & 0 & 1 & 1 & 0 & -2 & 0 & 0 & 1 & 0 & 1 & 1 & 1 & 1 & 1 & 1 & 0 & 1 & 1 & 1 & 0 \\
\hline$(-1,1,-1)$ & 0 & 1 & 0 & 1 & 0 & -2 & 0 & 1 & 1 & 1 & 0 & 1 & 0 & 1 & 1 & 1 & 0 & 1 & 1 & 1 \\
\hline$(-1,-1,1)$ & 0 & 0 & 1 & 1 & 0 & 0 & -2 & 1 & 1 & 0 & 1 & 1 & 1 & 0 & 1 & 1 & 1 & 1 & 0 & 1 \\
\hline$(-1,-1,-1)$ & 0 & 0 & 0 & 0 & 1 & 1 & 1 & -2 & 1 & 0 & 1 & 1 & 0 & 1 & 1 & 1 & 1 & 1 & 1 & 0 \\
\hline$(\tau-1, \tau, 0)$ & 1 & 1 & 0 & 1 & 0 & 1 & 1 & 1 & -2 & 1 & 0 & 1 & 1 & 1 & 0 & 0 & 1 & 1 & 0 & 0 \\
\hline$(1-\tau, \tau, 0)$ & 1 & 1 & 1 & 1 & 1 & 1 & 0 & 0 & 1 & -2 & 1 & 0 & 0 & 0 & 1 & 1 & 1 & 1 & 0 & 0 \\
\hline$(1-\tau,-\tau, 0)$ & 1 & 1 & 1 & 0 & 1 & 0 & 1 & 1 & 0 & 1 & -2 & 1 & 0 & 0 & 1 & 1 & 0 & 0 & 1 & 1 \\
\hline$(\tau-1,-\tau, 0)$ & 0 & 0 & 1 & 1 & 1 & 1 & 1 & 1 & 1 & 0 & 1 & -2 & 1 & 1 & 0 & 0 & 0 & 0 & 1 & 1 \\
\hline$(\tau, 0,1-\tau)$ & 1 & 1 & 1 & 1 & 1 & 0 & 1 & 0 & 1 & 0 & 0 & 1 & -2 & 1 & 0 & 1 & 0 & 1 & 1 & 0 \\
\hline$(\tau, 0, \tau-1)$ & 1 & 1 & 1 & 0 & 1 & 1 & 0 & 1 & 1 & 0 & 0 & 1 & 1 & -2 & 1 & 0 & 1 & 0 & 0 & 1 \\
\hline$(-\tau, 0, \tau-1)$ & 0 & 1 & 0 & 1 & 1 & 1 & 1 & 1 & 0 & 1 & 1 & 0 & 0 & 1 & -2 & 1 & 1 & 0 & 0 & 1 \\
\hline$(-\tau, 0,1-\tau)$ & 1 & 0 & 1 & 1 & 0 & 1 & 1 & 1 & 0 & 1 & 1 & 0 & 1 & 0 & 1 & -2 & 0 & 1 & 1 & 0 \\
\hline$(0, \tau-1, \tau)$ & 1 & 0 & 1 & 1 & 1 & 0 & 1 & 1 & 1 & 1 & 0 & 0 & 0 & 1 & 1 & 0 & -2 & 1 & 0 & 1 \\
\hline$(0, \tau-1,-\tau)$ & 0 & 1 & 1 & 0 & 1 & 1 & 1 & 1 & 1 & 1 & 0 & 0 & 1 & 0 & 0 & 1 & 1 & -2 & 1 & 0 \\
\hline$(0,1-\tau,-\tau)$ & 1 & 1 & 0 & 1 & 1 & 1 & 0 & 1 & 0 & 0 & 1 & 1 & 1 & 0 & 0 & 1 & 0 & 1 & -2 & 1 \\
\hline$(0,1-\tau, \tau)$ & 1 & 1 & 1 & 1 & 0 & 1 & 1 & 0 & 0 & 0 & 1 & 1 & 0 & 1 & 1 & 0 & 1 & 0 & 1 & -2 \\
\hline
\end{tabular}




\section{REFERENCES}

[Ba96] W. Barth, Two projective surfaces with many nodes, admitting the symmetries of the icosahedron, J. Alg. Geom. 5 (1996), 173-186.

[Bu91] H. Burkhardt, Untersuchungen aus dem Gebiete der hyperelliptischen Modulfunctionen, Math. Ann. 38 (1891), 161-224.

[CP10] I. Cheltsov, J. Park, Sextic double solids, Cohomological and Geometric Approaches to Rationality Problems, Progress in Mathematics 282 (2010), 75-132.

[CPS16] I. Cheltsov, V. Przyjalkowski, C. Shramov, Quartic double solids with icosahedral symmetry, European J. of Math. 2 (2016), no. 1, 96-119.

[CS14] I. Cheltsov, C. Shramov, Five embeddings of one simple group, Trans. of the AMS 366 (2014), 1289-1331.

[CS15] I. Cheltsov, C. Shramov, Cremona groups and the icosahedron, CRC Press, 2015.

[Co06] A. Coble, An invariant condition for certain automorphic algebraic forms, Amer. J. Math. 28 (1906), 333-366.

[Co00] A. Corti, Singularities of linear systems and 3-fold birational geometry, L.M.S. Lecture Note Series $\mathbf{2 8 1}$ (2000), 259-312.

$\left[\mathrm{C}^{+}\right]$J. Conway, R. Curtis, S. Norton, R. Parker, R. Wilson, Atlas of finite groups, Clarendon Press, Oxford, 1985.

[En99] S. Endraß, On the divisor class group of double solids, Manuscripta Mathematica 99 (1999), 341-358.

[GAP] The GAP Group, GAP — Groups, Algorithms, and Programming, Version 4.7.4; 2014.

[Hu96] B. Hunt, The geometry of some special arithmetic quotients, Lecture Notes in Mathematics, 1637. Berlin: Springer, 1996.

[IP99] V. Iskovskikh, Yu. Prokhorov, Fano Varieties, Encyclopaedia of Mathematical Sciences 47 (1999), Springer, Berlin.

[JR97] D. Jaffe, D. Ruberman, A sextic surface cannot have 66 nodes, J. Alg. Geom. 6:1 (1997), 151-168.

[JS-BV90] A. de Jong, N. Shepherd-Barron, A. Van de Ven, On the Burkhardt quartic, Math. Ann. 286 (1990), 309-328.

[Ka11] A.-S. Kaloghiros, The defect of Fano 3-folds, J. Alg. Geom. 20:1 (2011), 127-149.

$[\mathrm{Me} 04]$ M. Mella, Birational geometry of quartic 3-folds. II. The importance of being $\mathbb{Q}$-factorial, Math. Ann. 330 (2004), 107-126.

[Pr13] Yu. Prokhorov, G-Fano threefolds, I, II, Adv. Geom. 13 (2013), no. 3, 389-418, 419-434.

[Pr17] Yu. Prokhorov, On the number of singular points of factorial terminal Fano threefolds, Mathematical Notes, 2017, 101:6, 1068-1073.

[PS16] V. Przyjalkowski, C. Shramov, Double quadrics with large automorphism groups, Proc. Steklov Inst. Math., 294 (2016), 154-175.

[To35] J. Todd, On a quartic primal with forty-five nodes, in space of four dimensions, Q. J. Math. 7 (1935), $168-174$.

Ivan Cheltsov

School of Mathematics, The University of Edinburgh.

National Research University Higher School of Economics, Russian Federation.

I.Cheltsov@ed.ac.uk

Victor Przyjalkowski

Steklov Mathematical Institute of Russian Academy of Sciences, Moscow, Russia.

National Research University Higher School of Economics, Russian Federation.

victorprz@mi.ras.ru, victorprz@gmail.com

Constantin Shramov

Steklov Mathematical Institute of Russian Academy of Sciences, Moscow, Russia. National Research University Higher School of Economics, Russian Federation.

costya.shramov@gmail.com 